Article

\title{
Fruit Yield and Physicochemical Quality Evaluation of Hybrid and Grafted Field-Grown Muskmelon in Pennsylvania
}

\author{
Elsa Sánchez ${ }^{1, *}$, Robert Pollock $^{2}$, Timothy Elkner ${ }^{3}$, Thomas Butzler $^{4}$ and Francesco Di Gioia ${ }^{1}$ (D) \\ 1 Department of Plant Sciences, Pennsylvania State University, University Park, PA 16802, USA; fxd92@psu.edu \\ 2 Penn State Extension, Indiana County, Indiana, PA 15701, USA; rcp3@psu.edu \\ 3 Penn State Extension, Lancaster County, Lancaster, PA 17601, USA; tee2@psu.edu \\ 4 Penn State Extension, Clinton County, Lock Haven, PA 17745, USA; tmb124@psu.edu \\ * Correspondence: ess11@psu.edu
}

\section{check for}

updates

Citation: Sánchez, E.; Pollock, R.; Elkner, T.; Butzler, T.; Di Gioia, F. Fruit Yield and Physicochemical Quality Evaluation of Hybrid and Grafted Field-Grown Muskmelon in Pennsylvania. Horticulturae 2021, 7, 69. https://doi.org/10.3390/ horticulturae7040069

Academic Editors:

Federica Caradonia and Douglas D. Archbold

Received: 5 February 2021

Accepted: 1 April 2021

Published: 3 April 2021

Publisher's Note: MDPI stays neutral with regard to jurisdictional claims in published maps and institutional affiliations.

Copyright: (C) 2021 by the authors. Licensee MDPI, Basel, Switzerland. This article is an open access article distributed under the terms and conditions of the Creative Commons Attribution (CC BY) license (https:/ / creativecommons.org/licenses/by/ $4.0 /)$.

\begin{abstract}
Selecting vegetable cultivars suitable to local environmental conditions and with quality traits desired by the evolving market and consumer needs is an important production decision farmers face annually. As seed companies continue to expand their offerings of new cultivars and rootstocks, selecting the best cultivar and/or scion/rootstock combination can be challenging for farmers. Land-grant universities, through their integrated research and extension programs, can provide an unbiased, science-based evaluation of the available cultivar and rootstock options to assist farmers in making this important selection. A two-year study was conducted to evaluate 20 hybrid cultivars and two grafted entries of muskmelons at three locations in Pennsylvania in 2018 and 2019 to provide farmers with science-based recommendations focused on fruit yield and physicochemical quality characteristics. Most cultivars did not differ in fruit yields from the standard "Aphrodite". "Sugar Cube" produced more, smaller sized melons than "Aphrodite". However, the combination of the soluble solids concentration, flesh $\mathrm{pH}$, and titratable acidity values was not as favorable, indicating that consumer preference may be lower for "Sugar Cube" than for other cultivars. Yield from grafted entries was not different from the non-grafted "Aphrodite"; although, biotic and abiotic stressors favoring the use of grafting were not present throughout the study. Physicochemical evaluation of the combination of "Aphrodite" scion and "Flexifort" rootstock was more favorable than "Aphrodite/RS841" and non-grafted "Aphrodite". This combination may be desirable even in the absence of yield stressors.
\end{abstract}

Keywords: vegetable variety; cultivar evaluation; vegetable grafting; rootstock selection; fruit quality; cantaloupe; Cucumis melo

\section{Introduction}

Muskmelons (Cucumis melo L.) are a common component of diversified vegetable operations in Pennsylvania and are grown on about $14 \%$ of farms, primarily for fresh markets [1]. The increasing demand for local fresh high-quality produce is resulting in vegetable farmers in the northeastern U.S. diversifying their operations and increasing the seasonal availability of fresh fruits and vegetables [2]. Muskmelon and other cucurbits are an important rotational crop, primarily grown in the open-field during the springsummer season using raised-bed plasticulture systems equipped with drip-irrigation. In the northeastern USA, the growing season for warm-season vegetables like muskmelon is relatively short, starting in mid-May or early-June and ending with harvest in JulyAugust. Cultivar selection is critical for the success of this crop, especially considering the unpredictable weather conditions and rainfall patterns, changing from season to season. Numerous muskmelon cultivars are commercially available, and deciding which to grow is an important managerial decision. Additionally, this decision must be considered annually, as the market life cycle of individual cultivars is often short, and new ones are continuously added to the available offerings [3]. Vegetable and fruit farmers ranked cultivar selection as 
third in importance from a list of vegetable production activities in a 2019 survey $(n=110)$, behind irrigation management and crop rotation [4].

Additionally, an increasing number of muskmelon farmers are considering the use of grafted plants. They need assistance determining whether grafting is a good fit for their farms and with selecting rootstocks from those currently available on the market. Over the last few decades, with the phase-out of methyl bromide, the use of vegetable grafting is becoming more common in the USA [5], as it is increasingly proposed as a biological solution for addressing diseases caused by soilborne pathogens, as well as for managing abiotic stressors, including cold, heat, flooding, salinity, and mineral excess, or the combination of multiple stressors [6-10]. Despite these benefits, the adoption of vegetable grafting is currently limited in Pennsylvania, especially for cucurbit crops. This may be partly due to the concern that vegetable grafting may delay maturity and negatively impact fruit quality $[8,11,12]$, which is particularly important with muskmelon, especially for local markets. For muskmelons, flavor and texture are positively correlated with consumer preference [13]. Consumer preference and the overall eating quality of muskmelons have been linked to the physicochemical traits of the fruit [14]. There is a need to understand if there are new cultivars that produce higher yields and fruit quality, and if the use of grafted plants provides benefits or drawbacks in terms of fruit yield and quality under the pedoclimatic conditions typical of the U.S. mid-Atlantic region.

Through their research and extension programs, land-grant universities provide farmers an unbiased evaluation of cultivars and allied technologies, such as vegetable grafting. A reliable evaluation requires conducting field evaluations at multiple locations representative of the commercial production area, possibly repeated over a minimum of two seasons, while following the standard agronomic practices used by local farmers [15-19]. Using such protocols, it is possible to compare open-pollinated, hybrid, and/or grafted cultivars by measuring plant characteristics, yield performances, and/or quality of the harvested products, which are the main parameters farmers use for assessing the suitability of a cultivar or rootstock for their farms.

The goal of this research was to provide farmers with recommendations for commercially available muskmelon cultivars and grafted plant combinations by evaluating the yield components and physicochemical traits of the fruit across three locations representative of the main Pennsylvania vegetable production areas for two growing seasons. Funding and time constraints limit the number of cultivar evaluations scientists can conduct. In the mid-Atlantic, university Extension educators and faculty members from the University of Delaware, University of Maryland, Rutgers the State University of New Jersey, Pennsylvania State University, University of Virginia, and University of West Virginia post research-based recommendations for farmers in a commercial vegetable production guide [20] used in all six states. Recommendations from this evaluation were also intended to be included in that production guide.

\section{Materials and Methods}

A two-year field study was conducted to evaluate 20 cultivars of muskmelon and two grafted combinations (Table 1) in three locations representative of western, central, and southeastern Pennsylvania. Cultivars were selected based on conversations with farmers and seed company representatives. The two rootstocks evaluated were interspecific hybrids between Cucurbita maxima Duch. (winter squash) and Cucurbita moschata Duch. (pumpkin), and were selected for high and moderate vigor for RS841 and Flexifort, respectively. Both rootstocks had resistance to several soilborne pathogens and root-knot nematodes; however, these issues were not present in this study. 
Table 1. Cultivars, rootstocks, and seed sources of muskmelons evaluated at three sites in Pennsylvania in 2018-2019.

\begin{tabular}{|c|c|c|c|c|c|c|c|}
\hline \multicolumn{8}{|c|}{ Seed Companies and Headquarters } \\
\hline Clifton Seed & Rupp & Sakata & Seedway LLC & Seminis & Syngenta & Enza Zaden & De Ruiter Seeds \\
\hline Faison, NC & Wauseon, $\mathrm{OH}$ & Morgan Hill, CA & Mifflinburg, PA & St. Louis, MO & Greensboro, NC & Salinas, CA & Creve Coeur, MO \\
\hline \multicolumn{6}{|c|}{ Muskmelon cultivars } & \multicolumn{2}{|c|}{ Rootstocks $^{\text {z }}$} \\
\hline $\begin{array}{l}\text { Carousel } \\
\text { Lani } \\
\text { Fiji }\end{array}$ & $\begin{array}{c}\text { Ambassador } \\
\text { Tirreno }\end{array}$ & $\begin{array}{c}\text { Atlantis } \\
\text { Avatar } \\
\text { Infinite Gold }\end{array}$ & $\begin{array}{c}\text { Afterglow } \\
\text { Aphrodite } \\
\text { Atlantis } \\
\text { Goddess } \\
\text { Minerva } \\
\text { Shockwave } \\
\text { Sugar Cube } \\
\text { Verona }\end{array}$ & Sun Blushed & $\begin{array}{c}\text { Accolade } \\
\text { Aphrodite } \\
\text { Ariel } \\
\text { Astound } \\
\text { Athena }\end{array}$ & Flexifort & RS841 \\
\hline
\end{tabular}

z "Aphrodite" scions were grafted to rootstocks. Grafted plants were produced and provided by Tri-Hishtil LLC (Mills River, NC) a commercial nursery specialized in producing grafted plants for the USA market.

While the study had the same objective in all three locations, planting and harvesting dates, as well as crop management practices, varied by location, as reported in Table 2, and were determined based on what was optimal at each location. At all locations, experimental units contained six plants, and were arranged in a randomized complete block design with four replications.

Table 2. Sites, production protocols, and dates used in an evaluation of hybrids and grafted cultivars in Pennsylvania in 2018 and 2019.

\begin{tabular}{|c|c|c|c|}
\hline Experimental Details & Western Site & Central Site & Southeastern Site \\
\hline Location & $\begin{array}{l}\text { Commercial farm, Indiana; lat. } \\
40^{\circ} 39^{\prime} 30.9^{\prime \prime} \mathrm{N} 79^{\circ} 16^{\prime} 05.4^{\prime \prime} \mathrm{W}\end{array}$ & $\begin{array}{c}\text { Pennsylvania State } \\
\text { University's Russell E. Larson } \\
\text { Research Center, Pennsylvania } \\
\text { Furnace; lat. } 40^{\circ} 42^{\prime} 45.04^{\prime \prime} \mathrm{N}, \\
\text { long } 77^{\circ} 57^{\prime} 12.44^{\prime \prime} \mathrm{W}\end{array}$ & $\begin{array}{c}\text { Pennsylvania State } \\
\text { University"s Southeast } \\
\text { Research and Extension } \\
\text { Center, Manheim; lat. } 40^{\circ} \\
07^{\prime} 05.11^{\prime \prime} \mathrm{N} \text {, long. } 76^{\circ} \\
25^{\prime} 45.69^{\prime \prime} \mathrm{W}\end{array}$ \\
\hline Planting dates & 30 May 2018; 27 May 2019 & 30 May 2018; 11 June 2019 & 15 May 2018; 06 June 2019 \\
\hline Nitrogen rate & $128 \mathrm{~kg} /$ ha applied pre-plant & $\begin{array}{l}112 \mathrm{~kg} / \text { ha applied; } 60 \% \\
\text { applied pre-plant, } 40 \% \\
\text { fertigated throughout the } \\
\text { growing season }\end{array}$ & $\begin{array}{l}\text { None applied pre-plant; } \\
140 \mathrm{~kg} / \text { ha fertigated } \\
\text { throughout the growing } \\
\text { season }\end{array}$ \\
\hline $\begin{array}{l}\text { Phosphorus and potassium } \\
\text { fertility }\end{array}$ & $\begin{array}{l}54 \mathrm{~kg} / \mathrm{ha} \text { P applied pre-plant; } \\
104 \mathrm{~kg} / \text { ha K applied pre-plant }\end{array}$ & $\begin{array}{l}\text { P and K were not applied } \\
\text { based on soil test results }\end{array}$ & $112 \mathrm{~kg} / \mathrm{ha}$ applied pre-plant \\
\hline In-row spacing & $0.38 \mathrm{~m}$ & $0.61 \mathrm{~m}$ & $0.61 \mathrm{~m}$ \\
\hline $\begin{array}{l}\text { Row centers } \\
\text { Irrigation }\end{array}$ & $\begin{array}{l}\qquad .7 \mathrm{~m} \\
\text { Drip irrigation system } \\
\text { installed, but not used }\end{array}$ & $\begin{array}{c}3.7 \mathrm{~m} \\
\text { Drip irrigation used to } \\
\text { supplement rainfall to } \\
\text { provide } \sim 103 \mathrm{~m}^{3} \text { to } 155 \mathrm{~m}^{3} \text { of } \\
\text { water weekly }\end{array}$ & $\begin{array}{c}3.7 \mathrm{~m} \\
\text { Drip irrigation used to } \\
\text { supplement rainfall to } \\
\text { provide } \sim 103 \mathrm{~m}^{3} \text { to } 155 \mathrm{~m}^{3} \text { of } \\
\text { water weekly }\end{array}$ \\
\hline Harvest dates & $\begin{array}{l}31 \text { July-28 August 2018; } \\
30 \text { July-19 August } 2019\end{array}$ & $\begin{array}{c}\text { 4-29 August 2018; } \\
\text { 4 August-16 September } 2019\end{array}$ & $\begin{array}{c}\text { 13-July } 13 \text { August 2018; } \\
\text { 5-30 August } 2019\end{array}$ \\
\hline
\end{tabular}

At all sites, four- or five-week-old transplants were planted into a plasticulture system with raised beds, a single drip irrigation line (T-Tape model 508-12-450, John Deere, Moline, IL, USA), and embossed black plastic mulch (1 mil, Wrap Bros., Chicago, IL, USA). Pest management practices followed the 2018 Commercial Vegetable Production Recommendation guide [20].

Melons were harvested, counted, and weighed as individual fruit reached the full slip stage, when fruit easily separated from the vine by hand, and were categorized as 
marketable or unmarketable. Melons were considered marketable when they were fullsized, the rind had developed netting, they were a normal color for the cultivar, and they were free of cracks or other damage and disease.

At the central site only, postharvest physicochemical evaluations were conducted on the marketable fruit of 14 cultivars and the two grafted combinations. Within $48 \mathrm{~h}$ of harvest, two fruit per experimental unit were quartered, the placenta and rind were discarded, and the soluble solids concentration (SSC) was averaged from near the blossom and stem ends and the middle of the mesocarp with an analog handheld refractometer (Atago ATC-1, Bellevue, WA, USA). The fresh weight of $125 \mathrm{~g}$ mesocarp samples was recorded. Samples were then oven-dried at $\sim 66^{\circ} \mathrm{C}$ for $\sim 10$ days until constant weight and reweighed to determine the fruit dry matter content. Fresh mesocarp subsamples $(\sim 100 \mathrm{~g})$ were cut into cubes and stored at $-20^{\circ} \mathrm{C}$ until the analysis of flesh $\mathrm{pH}$ and titratable acidity (TA) was performed. Thawed tissue samples were juiced with $150 \mathrm{~mL}$ of distilled water and filtered through a coffee filter $(6.35 \times 8.25 \mathrm{~cm}$; Wegmans; Rochester, NY, USA). Fruit pH was determined with a table-top meter (Mini-titrator HI84532; Hanna Instruments; Smithfield, OR). Titratable acidity was measured by titrating to $\mathrm{pH} 8.2$ with $0.1 \mathrm{M} \mathrm{NaOH}$ and was reported as percent malic acid.

We were advised by a statistical consultant on the statistical analysis used in this research. Visual inspection of the yield data indicated a three-way interaction between site, year, and cultivar; therefore, data were analyzed by site. Yield and physicochemical properties data were analyzed using the mixed procedure and means were compared at $P<0.05$ using the "PDIFF" option to compare all means and adjusted using Tukey's honestly significant difference for multiple comparisons among means (SAS 9.4; Cary, NC, USA). When year-cultivar interactions were significant, means were compared within each year ( $P$-values available as Supplementary Tables S1 and S2).

\section{Results and Discussion}

\subsection{Yield}

Using a standard makes results more meaningful for farmers as it provides a reference point for comparison [21]. "Aphrodite" was used as the standard to which all other cultivars were compared because farmers told us this was the main cultivar they grow.

\subsubsection{Western Site}

In 2018, the mean number of marketable melons ranged from 0.00 to 1.92 per plant (Table 3). "Lani", "Shockwave", and "Fiji" produced fewer melons than "Aphrodite" (1.00 per plant). All other cultivars did not differ from "Aphrodite". In 2019, the range was similar at 0.00 to 1.88 per plant. "Sugar Cube", "Tirreno", "Carousel", "Astound", "Goddess", and "Athena" produced more melons than "Aphrodite" (0.29 per plant). All other cultivars did not differ from "Aphrodite".

In 2018, the mean weight of marketable melons ranged from $0.00 \mathrm{~kg}$ to $4.04 \mathrm{~kg}$ per plant (Table 3$)$. Weights from "Infinite Gold" (0.20 kg per plant),"Fiji" (0.00 kg per plant), "Lani" (0.00 kg per plant), and "Shockwave" (0.00 kg per plant) were lower than from "Aphrodite" (2.62 kg per plant). All other cultivars did not differ from "Aphrodite". In 2019, the range was smaller, from $0.00 \mathrm{~kg}$ to $1.86 \mathrm{~kg}$ per plant. Mean weights from "Carousel" and "Tirreno" were higher than from "Aphrodite" ( $0.65 \mathrm{~kg}$ per plant). All other cultivars did not differ from "Aphrodite".

In 2018, the mean number of unmarketable melons ranged from 0.08 to 1.29 per plant (Table 4). All cultivars did not differ from "Aphrodite" ( 0.67 per plant). In 2019, the range was similar, at 0.00 to 1.21 per plant. "Shockwave" and "Infinite Gold" produced more unmarketable melons than "Aphrodite" (0.00 per plant). All other cultivars did not differ from "Aphrodite". 
Table 3. Mean marketable fruit number ${ }^{\mathrm{z}}$ and weight per plant of 20 muskmelon cultivars and two grafted entries evaluated in western Pennsylvania in 2018 and 2019.

\begin{tabular}{ccccc}
\hline \multirow{2}{*}{ Cultivar } & \multicolumn{2}{c}{2018} & \multicolumn{2}{c}{2019} \\
\cline { 2 - 5 } & Number & Weight (kg) & Number & Weight (kg) \\
\hline Accolade & $0.75 \mathrm{~b}-\mathrm{f}$ y & $1.79 \mathrm{~b}-\mathrm{f}$ & $0.29 \mathrm{ef}$ & $0.78 \mathrm{c}-\mathrm{h}$ \\
Afterglow & $1.29 \mathrm{a}-\mathrm{d}$ & $2.66 \mathrm{a}-\mathrm{d}$ & $0.21 \mathrm{f}$ & $0.37 \mathrm{e}-\mathrm{h}$ \\
Aphrodite & $1.00 \mathrm{a}-\mathrm{e}$ & $2.62 \mathrm{a}-\mathrm{d}$ & $0.29 \mathrm{ef}$ & $0.65 \mathrm{c}-\mathrm{h}$ \\
Aphrodite/Flexifort & $1.00 \mathrm{a}-\mathrm{e}$ & $3.23 \mathrm{abc}$ & $0.46 \mathrm{c}-\mathrm{f}$ & $0.93 \mathrm{~b}-\mathrm{h}$ \\
Aphrodite/RS48 & $1.21 \mathrm{a}-\mathrm{d}$ & $4.04 \mathrm{a}$ & $0.17 \mathrm{f}$ & $0.39 \mathrm{e}-\mathrm{h}$ \\
Ariel & $1.08 \mathrm{a}-\mathrm{e}$ & $2.62 \mathrm{a}-\mathrm{d}$ & $0.50 \mathrm{c}-\mathrm{f}$ & $0.98 \mathrm{~b}-\mathrm{h}$ \\
Astound & $1.46 \mathrm{ab}$ & $3.28 \mathrm{abc}$ & $0.96 \mathrm{bc}$ & $1.51 \mathrm{abc}$ \\
Athena & $0.63 \mathrm{~b}-\mathrm{f}$ & $1.43 \mathrm{~b}-\mathrm{f}$ & $0.84 \mathrm{bcd}$ & $1.50 \mathrm{a}-\mathrm{d}$ \\
Atlantis & $1.17 \mathrm{a}-\mathrm{d}$ & $2.67 \mathrm{a}-\mathrm{d}$ & $0.75 \mathrm{~b}-\mathrm{e}$ & $1.35 \mathrm{a}-\mathrm{e}$ \\
Avatar & $0.79 \mathrm{~b}-\mathrm{f}$ & $2.84 \mathrm{a}-\mathrm{d}$ & $0.46 \mathrm{c}-\mathrm{f}$ & $1.15 \mathrm{~b}-\mathrm{g}$ \\
Carousel & $1.33 \mathrm{ab}$ & $3.33 \mathrm{abc}$ & $1.21 \mathrm{~b}$ & $2.20 \mathrm{a}$ \\
Fiji & $0.00 \mathrm{f}$ & $0.00 \mathrm{f}$ & $0.08 \mathrm{f}$ & $0.11 \mathrm{gh}$ \\
Goddess & $0.75 \mathrm{~b}-\mathrm{f}$ & $1.69 \mathrm{~b}-\mathrm{f}$ & $0.88 \mathrm{bc}$ & $1.61 \mathrm{abc}$ \\
Infinite Gold & $0.08 \mathrm{ef}$ & $0.20 \mathrm{ef}$ & $0.08 \mathrm{f}$ & $0.14 \mathrm{gh}$ \\
Lani & $0.00 \mathrm{f}$ & $0.00 \mathrm{f}$ & $0.00 \mathrm{f}$ & $0.00 \mathrm{~h}$ \\
Minerva & $0.58 \mathrm{~b}-\mathrm{f}$ & $1.40 \mathrm{~b}-$ & $0.29 \mathrm{ef}$ & $0.80 \mathrm{c}-\mathrm{h}$ \\
Shockwave & $0.00 \mathrm{f}$ & $0.00 \mathrm{f}$ & $0.33 \mathrm{def}$ & $0.47 \mathrm{~d}-\mathrm{h}$ \\
Sugar Cube & $1.92 \mathrm{a}$ & $1.86 \mathrm{a}-$ & $1.88 \mathrm{a}$ & $1.19 \mathrm{a}-\mathrm{f}$ \\
Sun Blushed & $0.38 \mathrm{def}$ & $0.86 \mathrm{de}$ & $0.17 \mathrm{f}$ & $0.29 \mathrm{fgh}$ \\
Tirreno & $1.17 \mathrm{a}-\mathrm{d}$ & $2.51 \mathrm{a}-\mathrm{e}$ & $1.25 \mathrm{~b}$ & $1.86 \mathrm{ab}$ \\
Verona & $0.88 \mathrm{~b}-\mathrm{f}$ & $2.91 \mathrm{a}-\mathrm{d}$ & $0.33 \mathrm{def}$ & $0.90 \mathrm{~b}-\mathrm{h}$
\end{tabular}

${ }^{\mathrm{z}}$ Values are the mean of four replications; data were analyzed using the mixed procedure and means were separated using PDIFF; values followed by different letters within a column are significantly different at $P<0.05$; "Aphrodite" was considered the standard. ${ }^{y}$ Experimental units consisted of six plants.

Table 4. Mean unmarketable fruit number ${ }^{\mathrm{z}}$ and weight per plant of 20 muskmelon cultivars and two grafted entries evaluated in western Pennsylvania in 2018 and 2019.

\begin{tabular}{ccccc}
\hline \multirow{2}{*}{ Cultivar } & \multicolumn{2}{c}{2018} & \multicolumn{2}{c}{$\mathbf{2 0 1 9}$} \\
\cline { 2 - 5 } & Number & Weight (kg) & Number & Weight (kg) \\
\hline Accolade & $0.33 \mathrm{bc}$ & $0.52 \mathrm{cde}$ & $0.13 \mathrm{~b}$ & $0.09 \mathrm{~b}$ \\
Afterglow & $0.54 \mathrm{abc}$ & $0.84 \mathrm{~b}-\mathrm{e}$ & $0.00 \mathrm{~b}$ & $0.00 \mathrm{~b}$ \\
Aphrodite & $0.67 \mathrm{abc}$ & $1.12 \mathrm{a}-\mathrm{e}$ & $0.00 \mathrm{~b}$ & $0.00 \mathrm{~b}$ \\
Aphrodite/Flexifort & $0.42 \mathrm{bc}$ & $1.29 \mathrm{a}-\mathrm{e}$ & $0.13 \mathrm{~b}$ & $0.18 \mathrm{~b}$ \\
Aphrodite/RS48 & $0.25 \mathrm{bc}$ & $0.66 \mathrm{~b}-\mathrm{e}$ & $0.04 \mathrm{~b}$ & $0.07 \mathrm{~b}$ \\
Ariel & $0.38 \mathrm{bc}$ & $0.82 \mathrm{~b}-\mathrm{e}$ & $0.08 \mathrm{~b}$ & $0.08 \mathrm{~b}$ \\
Astound & $0.29 \mathrm{bc}$ & $0.46 \mathrm{cde}$ & $0.00 \mathrm{~b}$ & $0.00 \mathrm{~b}$ \\
Athena & $0.21 \mathrm{bc}$ & $0.35 \mathrm{de}$ & $0.04 \mathrm{~b}$ & $0.03 \mathrm{~b}$ \\
Atlantis & $0.08 \mathrm{c}$ & $0.21 \mathrm{e}$ & $0.00 \mathrm{~b}$ & $0.00 \mathrm{~b}$ \\
Avatar & $0.08 \mathrm{c}$ & $0.20 \mathrm{e}$ & $0.04 \mathrm{~b}$ & $0.09 \mathrm{~b}$ \\
Carousel & $0.38 \mathrm{bc}$ & $0.88 \mathrm{~b}-\mathrm{e}$ & $0.00 \mathrm{~b}$ & $0.00 \mathrm{~b}$ \\
Fiji & $1.29 \mathrm{a}$ & $2.09 \mathrm{ab}$ & $0.17 \mathrm{~b}$ & $0.27 \mathrm{~b}$ \\
Goddess & $0.33 \mathrm{bc}$ & $0.62 \mathrm{~b}-\mathrm{e}$ & $0.33 \mathrm{~b}$ & $0.33 \mathrm{~b}$ \\
Infinite Gold & $0.54 \mathrm{abc}$ & $0.91 \mathrm{a}-\mathrm{e}$ & $1.17 \mathrm{a}$ & $2.01 \mathrm{a}$ \\
Lani & $0.54 \mathrm{abc}$ & $0.68 \mathrm{~b}-\mathrm{e}$ & $0.08 \mathrm{~b}$ & $0.08 \mathrm{~b}$ \\
Minerva & $0.17 \mathrm{c}$ & $0.34 \mathrm{de}$ & $0.21 \mathrm{~b}$ & $0.24 \mathrm{~b}$ \\
Shockwave & $0.46 \mathrm{abc}$ & $0.98 \mathrm{~b}-\mathrm{e}$ & $1.21 \mathrm{a}$ & $1.73 \mathrm{a}$ \\
Sugar Cube & $0.42 \mathrm{bc}$ & $0.38 \mathrm{cde}$ & $0.00 \mathrm{~b}$ & $0.00 \mathrm{~b}$ \\
Sun Blushed & $1.04 \mathrm{ab}$ & $1.89 \mathrm{abc}$ & $0.00 \mathrm{~b}$ & $0.00 \mathrm{~b}$ \\
Tirreno & $0.63 \mathrm{abc}$ & $1.02 \mathrm{a}-\mathrm{e}$ & $0.04 \mathrm{~b}$ & $0.02 \mathrm{~b}$ \\
Verona & $0.29 \mathrm{bc}$ & $0.70 \mathrm{~b}-\mathrm{e}$ & $0.04 \mathrm{~b}$ & $0.03 \mathrm{~b}$ \\
\hline
\end{tabular}

${ }^{\mathrm{z}}$ Values are the mean of four replications; data were analyzed using the mixed procedure and means were separated using PDIFF; values followed by different letters within a column are significantly different at $P<0.05$; "Aphrodite" was considered the standard. ${ }^{y}$ Experimental units consisted of six plants. 
In 2018, the mean weight of unmarketable melons ranged from $0.20 \mathrm{~kg}$ to $2.09 \mathrm{~kg}$ per plant (Table 4). All cultivars did not differ from "Aphrodite" (1.12 kg per plant). In 2019, the range was $0.00 \mathrm{~kg}$ to $2.01 \mathrm{~kg}$ per plant. Mean unmarketable weight from "Infinite Gold" and "Shockwave" was higher than from "Aphrodite" (0.00 kg per plant). All other cultivars did not differ from "Aphrodite".

\subsubsection{Central Site}

In 2018, the mean number of marketable melons ranged from 0.00 to 3.96 per plant (Table 5). "Lani" produced more melons (3.96 per plant) than "Aphrodite" (1.25 per plant). All other cultivars did not differ from "Aphrodite". In 2019, the range was similar at 0.29 to 3.54 per plant. "Sugar Cube" and "Lani" produced more and "Shockwave", "Infinite Gold", "Goddess", and "Fiji" fewer melons than "Aphrodite" (1.79 per plant). All other cultivars did not differ from "Aphrodite".

Table 5. Mean marketable number ${ }^{\mathrm{z}}$ and weight per plant of 20 muskmelon cultivars and two grafted entries evaluated in central Pennsylvania in 2018 and 2019.

\begin{tabular}{ccccc}
\hline \multirow{2}{*}{ Cultivar } & \multicolumn{2}{c}{$\mathbf{2 0 1 8}$} & \multicolumn{2}{c}{$\mathbf{2 0 1 9}$} \\
\cline { 2 - 5 } & Number & Weight (kg) & Number & Weight (kg) \\
\hline Accolade & $1.90 \mathrm{a}-\mathrm{d}$ y & $4.76 \mathrm{abc}$ & $1.88 \mathrm{cde}$ & $3.53 \mathrm{a}-\mathrm{d}$ \\
Afterglow & $3.08 \mathrm{ab}$ & $6.53 \mathrm{ab}$ & $2.33 \mathrm{bc}$ & $3.82 \mathrm{a}-\mathrm{d}$ \\
Aphrodite & $1.25 \mathrm{bcd}$ & $3.38 \mathrm{a}-\mathrm{d}$ & $1.79 \mathrm{cde}$ & $4.74 \mathrm{abc}$ \\
Aphrodite/Flexifort & $1.38 \mathrm{bcd}$ & $4.93 \mathrm{abc}$ & $1.25 \mathrm{~d}-\mathrm{g}$ & $3.35 \mathrm{a}-\mathrm{d}$ \\
Aphrodite/RS48 & $1.17 \mathrm{bcd}$ & $3.71 \mathrm{a}-\mathrm{d}$ & $1.75 \mathrm{cde}$ & $5.26 \mathrm{a}$ \\
Ariel & $2.29 \mathrm{abc}$ & $6.07 \mathrm{ab}$ & $1.92 \mathrm{~b}-\mathrm{e}$ & $3.98 \mathrm{a}-\mathrm{d}$ \\
Astound & $2.21 \mathrm{abc}$ & $5.99 \mathrm{ab}$ & $2.29 \mathrm{bcd}$ & $4.72 \mathrm{abc}$ \\
Athena & $2.71 \mathrm{ab}$ & $4.49 \mathrm{abc}$ & $1.79 \mathrm{cde}$ & $3.70 \mathrm{a}-\mathrm{d}$ \\
Atlantis & $0.58 \mathrm{~cd}$ & $1.32 \mathrm{~cd}$ & $1.17 \mathrm{efg}$ & $2.71 \mathrm{cde}$ \\
Avatar & $1.63 \mathrm{bcd}$ & $5.91 \mathrm{ab}$ & $1.67 \mathrm{cde}$ & $4.97 \mathrm{ab}$ \\
Carousel & $1.75 \mathrm{bcd}$ & $4.16 \mathrm{a}-\mathrm{d}$ & $1.38 \mathrm{c}-\mathrm{f}$ & $3.45 \mathrm{a}-\mathrm{d}$ \\
Fiji & $2.79 \mathrm{ab}$ & $5.73 \mathrm{ab}$ & $0.29 \mathrm{~g}$ & $0.44 \mathrm{f}$ \\
Goddess & $2.21 \mathrm{abc}$ & $5.04 \mathrm{abc}$ & $0.42 \mathrm{fg}$ & $0.66 \mathrm{ef}$ \\
Infinite Gold & $0.00 \mathrm{~d}$ & $0.00 \mathrm{~d}$ & $0.43 \mathrm{fg}$ & $0.61 \mathrm{ef}$ \\
Lani & $3.96 \mathrm{a}$ & $4.04 \mathrm{a}-\mathrm{d}$ & $2.96 \mathrm{ab}$ & $3.38 \mathrm{a}-\mathrm{d}$ \\
Minerva & $1.67 \mathrm{bc}$ & $5.30 \mathrm{abc}$ & $1.83 \mathrm{cde}$ & $4.45 \mathrm{a}-\mathrm{d}$ \\
Shockwave & $1.71 \mathrm{bcd}$ & $4.21 \mathrm{a}-\mathrm{d}$ & $0.50 \mathrm{fg}$ & $0.69 \mathrm{ef}$ \\
Sugar Cube & $2.20 \mathrm{abc}$ & $2.47 \mathrm{bcd}$ & $3.54 \mathrm{a}$ & $3.29 \mathrm{a}-\mathrm{d}$ \\
Sun Blushed & $1.96 \mathrm{a}-\mathrm{d}$ & $5.09 \mathrm{abc}$ & $1.29 \mathrm{c}-\mathrm{g}$ & $2.87 \mathrm{~b}-\mathrm{e}$ \\
Tirreno & $3.17 \mathrm{ab}$ & $6.72 \mathrm{a}$ & $1.38 \mathrm{c}-\mathrm{f}$ & $2.23 \mathrm{def}$ \\
Verona & $1.54 \mathrm{bcd}$ & $4.81 \mathrm{abc}$ & $1.83 \mathrm{cde}$ & $5.44 \mathrm{a}$
\end{tabular}

z Values are the mean of four replications; data were analyzed using the mixed procedure and means were separated using PDIFF; values followed by different letters within a column are significantly different at $P<0.05$; "Aphrodite" was considered the standard. y Experimental units consisted of six plants.

In 2018, the mean weight of marketable melons ranged from $0.00 \mathrm{~kg}$ to $6.72 \mathrm{~kg}$ per plant (Table 5). All cultivars did not differ from "Aphrodite" (3.38 kg per plant). In 2019, the range was $0.44 \mathrm{~kg}$ to $5.44 \mathrm{~kg}$ per plant. "Tirreno", "Shockwave", "Goddess", "Infinite Gold", and "Fiji" had lower marketable weights than "Aphrodite (4.74 kg per plant). All other cultivars did not differ from "Aphrodite".

In 2018, the mean number of unmarketable melons ranged from 0.00 to 1.17 per plant (Table 6). "Atlantis" produced more unmarketable melons than "Aphrodite" (0.50 per plant). All other cultivars did not differ from "Aphrodite". In 2019, the range was wider, at 0.00 to 2.17 per plant. "Fiji" and "Shockwave" produced more unmarketable melons than "Aphrodite" (0.08 per plant). All other cultivars did not differ from "Aphrodite". 
Table 6. Mean unmarketable fruit number ${ }^{\mathrm{z}}$ and weight per plant of 20 muskmelon cultivars and two grafted entries evaluated in central Pennsylvania in 2018 and 2019.

\begin{tabular}{ccccc}
\hline \multirow{2}{*}{ Cultivar } & \multicolumn{2}{c}{2018} & \multicolumn{2}{c}{ 2019 } \\
\cline { 2 - 5 } & Number & Weight (kg) & Number & Weight (kg) \\
\hline Accolade & $0.22 \mathrm{~b}$ & $0.63 \mathrm{~b}$ & $0.79 \mathrm{bc}$ & $0.14 \mathrm{~b}$ \\
Afterglow & $0.13 \mathrm{~b}$ & $0.17 \mathrm{~b}$ & $0.04 \mathrm{c}$ & $0.05 \mathrm{~b}$ \\
Aphrodite & $0.50 \mathrm{~b}$ & $1.32 \mathrm{ab}$ & $0.08 \mathrm{c}$ & $0.10 \mathrm{~b}$ \\
Aphrodite/Flexifort & $0.38 \mathrm{~b}$ & $0.55 \mathrm{~b}$ & $0.04 \mathrm{c}$ & $0.18 \mathrm{~b}$ \\
Aphrodite/RS48 & $0.25 \mathrm{~b}$ & $0.68 \mathrm{~b}$ & $0.21 \mathrm{c}$ & $0.59 \mathrm{~b}$ \\
Ariel & $0.04 \mathrm{~b}$ & $0.11 \mathrm{~b}$ & $0.00 \mathrm{c}$ & $0.00 \mathrm{~b}$ \\
Astound & $0.00 \mathrm{~b}$ & $0.00 \mathrm{~b}$ & $0.13 \mathrm{c}$ & $0.13 \mathrm{~b}$ \\
Athena & $0.08 \mathrm{~b}$ & $0.15 \mathrm{~b}$ & $0.13 \mathrm{c}$ & $0.20 \mathrm{~b}$ \\
Atlantis & $1.17 \mathrm{a}$ & $2.81 \mathrm{a}$ & $0.00 \mathrm{c}$ & $0.00 \mathrm{~b}$ \\
Avatar & $0.33 \mathrm{~b}$ & $1.21 \mathrm{~b}$ & $0.00 \mathrm{c}$ & $0.00 \mathrm{~b}$ \\
Carouse & $0.08 \mathrm{~b}$ & $0.27 \mathrm{~b}$ & $0.38 \mathrm{c}$ & $0.84 \mathrm{~b}$ \\
Fiji & $0.21 \mathrm{~b}$ & $0.00 \mathrm{~b}$ & $2.17 \mathrm{a}$ & $2.85 \mathrm{a}$ \\
Goddess & $0.50 \mathrm{~b}$ & $0.98 \mathrm{~b}$ & $0.38 \mathrm{c}$ & $0.55 \mathrm{~b}$ \\
Infinite Gold & $0.00 \mathrm{~b}$ & $0.00 \mathrm{~b}$ & $0.78 \mathrm{bc}$ & $0.80 \mathrm{~b}$ \\
Lani & $0.00 \mathrm{~b}$ & $0.00 \mathrm{~b}$ & $0.04 \mathrm{c}$ & $0.02 \mathrm{~b}$ \\
Minerva & $0.33 \mathrm{~b}$ & $0.92 \mathrm{~b}$ & $0.00 \mathrm{c}$ & $0.00 \mathrm{~b}$ \\
Shockwave & $0.00 \mathrm{~b}$ & $0.00 \mathrm{~b}$ & $1.75 \mathrm{ab}$ & $2.33 \mathrm{a}$ \\
Sugar Cube & $0.00 \mathrm{~b}$ & $0.00 \mathrm{~b}$ & $0.04 \mathrm{c}$ & $0.05 \mathrm{~b}$ \\
Sun Blushed & $0.21 \mathrm{~b}$ & $0.64 \mathrm{~b}$ & $0.13 \mathrm{c}$ & $0.16 \mathrm{~b}$ \\
Tirreno & $0.00 \mathrm{~b}$ & $0.00 \mathrm{~b}$ & $0.00 \mathrm{c}$ & $0.00 \mathrm{~b}$ \\
Verona & $0.25 \mathrm{~b}$ & $0.61 \mathrm{~b}$ & $0.17 \mathrm{c}$ & $0.11 \mathrm{~b}$
\end{tabular}

$\overline{\mathrm{z}}$ Values are the mean of four replications; data were analyzed using the mixed procedure and means were separated using PDIFF; values followed by different letters within a column are significantly different at $P<0.05$; "Aphrodite" was considered the standard. ${ }^{y}$ Experimental units consisted of six plants.

In 2018, the mean weight of unmarketable melons ranged from $0.00 \mathrm{~kg}$ to $2.81 \mathrm{~kg}$ per plant (Table 6). All cultivars did not differ from "Aphrodite" (1.32 kg per plant). In 2019, it ranged from $0.00 \mathrm{~kg}$ to $2.85 \mathrm{~kg}$ per plant. "Fiji" and "Shockwave" produced a higher mean unmarketable weight than "Aphrodite" ( $0.10 \mathrm{~kg}$ per plant). All other cultivars did not differ from "Aphrodite".

\subsubsection{Southeastern Site}

In 2018, the mean number of marketable melons ranged from 0.04 to 2.50 per plant (Table 7). "Sugar Cube" produced more melons than "Aphrodite" (1.25 per plant) and "Shockwave", "Lani", "Fiji", and "Infinite Gold" produced fewer. All other cultivars did not differ from "Aphrodite". In 2019, the range was 0.08 to 2.75 per plant. "Sugar Cube", "Astound", "Atlantis", "Athena", and "Carousel" produced more, and "Fiji", fewer melons than "Aphrodite" (1.04 per plant). All other cultivars did not differ from "Aphrodite".

In 2018, the mean weight of marketable melons ranged from $0.07 \mathrm{~kg}$ to $5.82 \mathrm{~kg}$ per plant (Table 7). "Shockwave", "Lani", "Fiji", and "Infinite Gold" produced a lower weight than "Aphrodite" (4.26 kg per plant). All other cultivars did not differ from "Aphrodite". In 2019, the range was $0.21 \mathrm{~kg}$ to $5.93 \mathrm{~kg}$ per plant. "Shockwave", "Goddess", "Infinite Gold", "Lani", and "Fiji" produced a lower weight than "Aphrodite" (3.97 kg per plant). All other cultivars did not differ from "Aphrodite".

In 2018, the mean number of unmarketable melons ranged from 0.00 to 1.58 per plant (Table 8$)$. "Goddess" produced more than "Aphrodite" (0.38 per plant). All other cultivars did not differ from "Aphrodite". In 2019, the range was 0.13 to 1.38 per plant. "Atlantis", "Infinite Gold", "Avatar", and "Fiji" produced fewer than "Aphrodite" (1.0 per plant). All other cultivars did not differ from "Aphrodite". 
Table 7. Mean marketable number ${ }^{\mathrm{z}}$ and weight per plant of 20 muskmelon cultivars and two grafted entries evaluated in southeastern Pennsylvania in 2018 and 2019.

\begin{tabular}{ccccc}
\hline \multirow{2}{*}{ Cultivar } & \multicolumn{2}{c}{2018} & \multicolumn{2}{c}{2019} \\
\cline { 2 - 5 } & Number & Weight (kg) & Number & Weight (kg) \\
\hline Accolade & $1.50 \mathrm{bc}$ y & $4.55 \mathrm{a}-\mathrm{d}$ & $1.63 \mathrm{~b}-\mathrm{e}$ & $4.29 \mathrm{a}-\mathrm{d}$ \\
Afterglow & $1.46 \mathrm{bc}$ & $4.05 \mathrm{a}-\mathrm{d}$ & $1.33 \mathrm{~b}-\mathrm{g}$ & $3.20 \mathrm{cde}$ \\
Aphrodite & $1.25 \mathrm{bc}$ & $4.26 \mathrm{a}-\mathrm{d}$ & $1.04 \mathrm{e}-\mathrm{i}$ & $3.97 \mathrm{a}-\mathrm{d}$ \\
Aphrodite/Flexifort & $1.17 \mathrm{bc}$ & $5.11 \mathrm{abc}$ & $0.63 \mathrm{hij}$ & $2.33 \mathrm{de}$ \\
Aphrodite/RS48 & $1.42 \mathrm{bc}$ & $5.49 \mathrm{ab}$ & $0.71 \mathrm{~g}-\mathrm{j}$ & $2.75 \mathrm{cde}$ \\
Ariel & $1.13 \mathrm{bc}$ & $3.48 \mathrm{bcd}$ & $1.25 \mathrm{c}-\mathrm{h}$ & $4.20 \mathrm{a}-\mathrm{d}$ \\
Astound & $1.58 \mathrm{bc}$ & $4.46 \mathrm{a}-\mathrm{d}$ & $1.88 \mathrm{~b}$ & $4.54 \mathrm{abc}$ \\
Athena & $1.75 \mathrm{~b}$ & $4.96 \mathrm{a}-\mathrm{d}$ & $1.75 \mathrm{bcd}$ & $4.47 \mathrm{abc}$ \\
Atlantis & $1.21 \mathrm{bc}$ & $3.78 \mathrm{bcd}$ & $1.83 \mathrm{bc}$ & $5.49 \mathrm{ab}$ \\
Avatar & $1.29 \mathrm{bc}$ & $5.82 \mathrm{a}$ & $1.42 \mathrm{~b}-\mathrm{f}$ & $5.66 \mathrm{ab}$ \\
Carousel & $1.29 \mathrm{bc}$ & $4.85 \mathrm{a}-\mathrm{d}$ & $1.75 \mathrm{bcd}$ & $5.93 \mathrm{a}$ \\
Fiji & $0.08 \mathrm{~d}$ & $0.18 \mathrm{e}$ & $0.08 \mathrm{j}$ & $0.21 \mathrm{f}$ \\
Goddess & $1.21 \mathrm{bc}$ & $3.21 \mathrm{~cd}$ & $0.50 \mathrm{ij}$ & $1.52 \mathrm{ef}$ \\
Infinite Gold & $0.04 \mathrm{~d}$ & $0.07 \mathrm{e}$ & $0.58 \mathrm{hij}$ & $1.42 \mathrm{ef}$ \\
Lani & $0.13 \mathrm{~d}$ & $0.18 \mathrm{e}$ & $0.83 \mathrm{f}-\mathrm{i}$ & $1.41 \mathrm{ef}$ \\
Minerva & $0.92 \mathrm{c}$ & $3.59 \mathrm{bcd}$ & $1.13 \mathrm{~d}-\mathrm{i}$ & $4.17 \mathrm{a}-\mathrm{d}$ \\
Shockwave & $0.21 \mathrm{~d}$ & $0.76 \mathrm{e}$ & $0.63 \mathrm{hij}$ & $1.59 \mathrm{ef}$ \\
Sugar Cube & $2.50 \mathrm{a}$ & $3.01 \mathrm{~d}$ & $2.75 \mathrm{a}$ & $3.66 \mathrm{bcd}$ \\
Sun Blushed & $1.17 \mathrm{bc}$ & $3.22 \mathrm{~cd}$ & $1.08 \mathrm{~d}-\mathrm{i}$ & $2.97 \mathrm{cde}$ \\
Tirreno & $1.33 \mathrm{bc}$ & $3.20 \mathrm{~cd}$ & $1.58 \mathrm{~b}-\mathrm{e}$ & $3.86 \mathrm{a}-\mathrm{d}$ \\
Verona & $1.08 \mathrm{bc}$ & $4.18 \mathrm{a}-\mathrm{d}$ & $1.17 \mathrm{c}-\mathrm{i}$ & $4.69 \mathrm{abc}$
\end{tabular}

${ }^{\mathrm{z}}$ Values are the mean of four replications; data were analyzed using a mixed procedure and means were separated using PDIFF; values followed by different letters within a column are significantly different at $P<0.05$; "Aphrodite" was considered the standard. ${ }^{y}$ Experimental units consisted of six plants.

Table 8. Mean unmarketable number ${ }^{\mathrm{z}}$ and weight per plant of 20 muskmelon cultivars and two grafted entries evaluated in Southeastern Pennsylvania in 2018 and 2019.

\begin{tabular}{ccccc}
\hline \multirow{2}{*}{ Cultivar } & \multicolumn{2}{c}{2018} & \multicolumn{2}{c}{2019} \\
\cline { 2 - 5 } & Number & Weight (kg) & Number & Weight (kg) \\
\hline Accolade & $0.33 \mathrm{bcd}$ y & $0.98 \mathrm{~b}-\mathrm{f}$ & $0.54 \mathrm{~d}-\mathrm{h}$ & $1.47 \mathrm{def}$ \\
Afterglow & $0.38 \mathrm{bcd}$ & $0.98 \mathrm{~b}-\mathrm{f}$ & $0.46 \mathrm{fgh}$ & $1.15 \mathrm{def}$ \\
Aphrodite & $0.38 \mathrm{bcd}$ & $1.66 \mathrm{~b}-\mathrm{f}$ & $1.00 \mathrm{a}-\mathrm{f}$ & $3.31 \mathrm{abc}$ \\
Aphrodite/Flexifort & $0.71 \mathrm{~b}$ & $2.71 \mathrm{ab}$ & $1.13 \mathrm{abc}$ & $4.45 \mathrm{a}$ \\
Aphrodite/RS48 & $0.46 \mathrm{bcd}$ & $1.96 \mathrm{bcd}$ & $1.04 \mathrm{a}-\mathrm{e}$ & $4.42 \mathrm{a}$ \\
Ariel & $0.63 \mathrm{bc}$ & $2.30 \mathrm{abc}$ & $0.58 \mathrm{c}-\mathrm{h}$ & $1.75 \mathrm{c}-\mathrm{f}$ \\
Astound & $0.38 \mathrm{bcd}$ & $1.08 \mathrm{~b}-\mathrm{f}$ & $0.67 \mathrm{~b}-\mathrm{h}$ & $1.66 \mathrm{def}$ \\
Athena & $0.42 \mathrm{bcd}$ & $1.31 \mathrm{~b}-\mathrm{f}$ & $0.75 \mathrm{~b}-\mathrm{g}$ & $1.66 \mathrm{c}-\mathrm{f}$ \\
Atlantis & $0.25 \mathrm{bcd}$ & $0.75 \mathrm{c}-\mathrm{f}$ & $0.42 \mathrm{gh}$ & $1.09 \mathrm{def}$ \\
Avatar & $0.42 \mathrm{bcd}$ & $1.84 \mathrm{~b}-\mathrm{e}$ & $0.13 \mathrm{~h}$ & $0.65 \mathrm{ef}$ \\
Carousel & $0.50 \mathrm{bcd}$ & $1.56 \mathrm{~b}-\mathrm{f}$ & $0.58 \mathrm{c}-\mathrm{h}$ & $1.96 \mathrm{cde}$ \\
Fiji & $0.13 \mathrm{~cd}$ & $0.11 \mathrm{ef}$ & $0.13 \mathrm{~h}$ & $0.31 \mathrm{f}$ \\
Goddess & $1.58 \mathrm{a}$ & $3.97 \mathrm{a}$ & $1.38 \mathrm{a}$ & $3.70 \mathrm{ab}$ \\
Infinite Gold & $0.00 \mathrm{~d}$ & $0.00 \mathrm{f}$ & $0.42 \mathrm{gh}$ & $1.14 \mathrm{def}$ \\
Lani & $0.17 \mathrm{bcd}$ & $0.28 \mathrm{def}$ & $0.79 \mathrm{~b}-\mathrm{g}$ & $1.27 \mathrm{def}$ \\
Minerva & $0.46 \mathrm{bcd}$ & $1.80 \mathrm{~b}-\mathrm{e}$ & $1.08 \mathrm{a}-\mathrm{d}$ & $3.98 \mathrm{ab}$ \\
Shockwave & $0.29 \mathrm{bcd}$ & $0.86 \mathrm{c}-\mathrm{f}$ & $0.79 \mathrm{~b}-\mathrm{g}$ & $2.05 \mathrm{cde}$ \\
Sugar Cube & $0.71 \mathrm{~b}$ & $0.85 \mathrm{c}-\mathrm{f}$ & $1.17 \mathrm{ab}$ & $1.58 \mathrm{def}$ \\
Sun Blushed & $0.58 \mathrm{bc}$ & $1.38 \mathrm{~b}-\mathrm{f}$ & $0.75 \mathrm{~b}-\mathrm{g}$ & $1.89 \mathrm{c}-\mathrm{f}$ \\
Tirreno & $0.46 \mathrm{bcd}$ & $1.18 \mathrm{~b}-\mathrm{f}$ & $0.50 \mathrm{e}-\mathrm{h}$ & $1.33 \mathrm{def}$ \\
Verona & $0.58 \mathrm{bc}$ & $2.42 \mathrm{abc}$ & $0.67 \mathrm{~b}-\mathrm{h}$ & $2.39 \mathrm{bcd}$ \\
\hline \hline
\end{tabular}

${ }^{z}$ Values are the mean of four replications; data were analyzed using the mixed procedure and means were separated using PDIFF; values followed by different letters within a column are significantly different at $P<0.05$; "Aphrodite" was considered the standard. ${ }^{y}$ Experimental units consisted of six plants. 
In 2018, the mean weight of unmarketable melons ranged from $0.00 \mathrm{~kg}$ to $3.97 \mathrm{~kg}$ per plant (Table 8). "Goddess" produced a higher weight than "Aphrodite" (1.66 kg per plant). All other cultivars did not differ from "Aphrodite". In 2019, the range was $0.31 \mathrm{~kg}$ to 4.45 kg per plant. "Astound", "Sugar Cube", "Accolade", "Tirreno", "Lani", "Afterglow", "Infinite Gold", "Atlantis", "Avatar", and "Fiji" produced lower weights than "Aphrodite" (3.31 kg per plant). All other cultivars did not differ from "Aphrodite".

\subsubsection{Yield Performance of Hybrid Cultivars}

Yields from most cultivars did not differ from "Aphrodite" over the 2 years and were comparable to muskmelon yields in other Pennsylvania studies [22,23]. Given that most cultivars did not produce different yields than "Aphrodite", farmers could base cultivar selection on other criteria, including disease resistance and fruit quality, without sacrificing yield. The ability to select cultivars based on disease resistance without forfeiting yield may be particularly advantageous for low-input and organic farmers, who have fewer options for disease management than conventional farmers.

"Sugar Cube" produced more melons than "Aphrodite" in four site-years. Additionally, the mean marketable fruit weight from "Sugar Cube" was not different than "Aphrodite", indicating that melons were generally smaller, but the number produced was larger. "Sugar Cube" was bred to be small in size to reduce food waste [24]. Due to its smaller size, it may be a good option for selling separately from other cultivars. Indeed, we have observed this cultivar for sale individually by cultivar name at farmers' markets in Pennsylvania.

"Infinite Gold" produced fewer melons and had a lower mean marketable weight than "Aphrodite" in four site-years. Days to maturity were longer for "Infinite Gold" than "Aphrodite" (85 days and 72 days, respectively), and the growing season ended before several "Infinite Gold" fruit were harvestable. "Shockwave" and "Fiji" produced a lower mean marketable weight in four site-years, and "Lani" in three site-years. When high marketable weights are desired other cultivars may be better options.

\subsubsection{Yield Performance of Grafted Cultivars}

Grafted cultivars did not result in higher marketable yields by number or weight compared to non-grafted "Aphrodite" in all three locations for both growing seasons, regardless of the rootstock used (Tables 3, 5 and 7). Grafting has been increasingly proposed and used as a tool to overcome soilborne pests and diseases [5,25], to address abiotic stresses [9,26,27], and to increase yield $[8,28]$. "Flexifort" has a high resistance to Fusarium oxysporum f.sp. melonis (Fom) races 0, 1, 2, and 1.2, intermediate resistance to Fusarium oxysporum $\mathrm{f}$. sp. radicis-cucumerinum (Forc), and a claimed tolerance to drought, heat, cold, and salinity stress, and, "RS841" has high resistance to Fusarium oxysporum f.sp. cucumerinum (Foc) races 1 and 2, Fom races $0,1,1.2$, and 2, Fusarium oxysporum f.sp. niveum (Fon) races 1 and 2, and Fusarium oxysporum f. sp. radicis-lycopersici (For), as well as intermediate resistance to Rhizoctonia solani, and Meloidogyne sp. [29,30]. However, disease and pest pressure, and these abiotic stresses were low or absent throughout this study. Therefore, while these findings differ from previous studies [31-34], results from this study may be explained by the absence of soilborne pests and pathogens and specific abiotic stressors over the 2 years and at the three locations of this study. These results suggest that in the presence of low or no soilborne pests and pathogens and abiotic stress conditions there is no yield advantage to using grafted plants under the Pennsylvania pedoclimatic conditions.

\subsection{Postharvest Fruit Physicochemical Properties}

Soluble solids concentration (SSC) is highly correlated with sugar concentration and is used as a measure of marketability [14,35]. SSC ranged from $7.79 \%$ for "Astound" to $11.06 \%$ for "Aphrodite" (Table 9). It was not different between cultivars; however, it was higher in 2019 , at $10.42 \%$, than in 2018 , at $8.58 \%$. The U.S.D.A.'s protocols for muskmelons for shipping point and market inspection include methods for measuring SSC to determine 
the internal quality [35]. Soluble solids concentration between $9 \%$ and $10.99 \%$ is reported as "good internal quality" and $11 \%$ or greater as "very good internal quality." Furthermore, SSC is reported to the "nearest half percent." Using these standards, melons had "good internal quality" in 2019, but not in 2018. Irrigation practices can influence muskmelon SSC, where applying higher amounts of water is correlated with lower values [36,37]. In our case, lower values in 2018 could be related to record high rainfall [38], particularly in July, August, and September, which likely lowered the sugar concentration (Table 10).

Table 9. Physiochemical quality properties of 14 muskmelon cultivars and two grafted entries evaluated in central Pennsylvania in 2018 and 2019.

\begin{tabular}{|c|c|c|c|c|c|c|}
\hline Year & 2018-2019 & 2018-2019 & 2018 & 2019 & 2018-2019 & 2018-2019 \\
\hline $\begin{array}{l}\text { Fruit Quality } \\
\text { Parameters }\end{array}$ & $\begin{array}{l}\text { SSC (soluble solids } \\
\text { concentration) }(\%)\end{array}$ & Flesh pH & TA (titratal & cidity) ${ }^{z}(\%)$ & SSC/TA $^{\text {y }}$ & $\begin{array}{c}\text { Juiciness (\% } \\
\text { Water Content) }\end{array}$ \\
\hline \multicolumn{7}{|l|}{ Cultivar } \\
\hline Accolade & $9.38 a^{x}$ & $7.00 \mathrm{a}-\mathrm{d}$ & $0.02907 \mathrm{a}$ & $0.03408 \mathrm{bcd}$ & $357.53 \mathrm{a}$ & $91.94 \mathrm{bc}$ \\
\hline Afterglow & $9.27 \mathrm{a}$ & $7.00 \mathrm{a}-\mathrm{d}$ & $0.02533 a$ & $0.03829 \mathrm{bcd}$ & $352.85 \mathrm{a}$ & $93.22 \mathrm{abc}$ \\
\hline Aphrodite & $11.06 \mathrm{a}$ & $7.07 \mathrm{a}-\mathrm{d}$ & $0.02357 \mathrm{a}$ & $0.03506 \mathrm{bcd}$ & $401.09 \mathrm{a}$ & $92.59 \mathrm{abc}$ \\
\hline Aphrodite/Flexifort & $9.91 \mathrm{a}$ & $7.33 \mathrm{a}$ & $0.02356 \mathrm{a}$ & $0.02173 \mathrm{~d}$ & $473.37 \mathrm{a}$ & $92.69 \mathrm{abc}$ \\
\hline Aphrodite/RS841 & $9.29 \mathrm{a}$ & $6.98 \mathrm{a}-\mathrm{d}$ & $0.02093 \mathrm{a}$ & $0.03410 \mathrm{bcd}$ & $378.88 \mathrm{a}$ & $92.70 \mathrm{abc}$ \\
\hline Ariel & $7.99 \mathrm{a}$ & $6.80 \mathrm{~d}$ & $0.02809 \mathrm{a}$ & $0.03866 \mathrm{bcd}$ & $243.61 \mathrm{a}$ & $94.65 \mathrm{a}$ \\
\hline Astound & $7.79 \mathrm{a}$ & $6.90 \mathrm{bcd}$ & $0.02050 \mathrm{a}$ & $0.03605 \mathrm{bcd}$ & $305.47 \mathrm{a}$ & $94.14 \mathrm{ab}$ \\
\hline Athena & $9.59 \mathrm{a}$ & $6.93 \mathrm{bcd}$ & $0.03448 \mathrm{a}$ & $0.03385 \mathrm{bcd}$ & $285.55 \mathrm{a}$ & $91.28 \mathrm{c}$ \\
\hline Atlantis & $9.38 \mathrm{a}$ & $6.97 \mathrm{a}-\mathrm{d}$ & $0.02946 \mathrm{a}$ & $0.02776 \mathrm{~cd}$ & $337.40 \mathrm{a}$ & $92.75 \mathrm{abc}$ \\
\hline Carousel & $10.05 \mathrm{a}$ & $6.85 \mathrm{~cd}$ & $0.03003 \mathrm{a}$ & $0.04660 \mathrm{~b}$ & $262.06 \mathrm{a}$ & $92.33 \mathrm{abc}$ \\
\hline Minerva & $9.37 \mathrm{a}$ & $7.07 \mathrm{a}-\mathrm{d}$ & $0.01468 \mathrm{a}$ & 0.04412 bc & $371.97 \mathrm{a}$ & $92.73 \mathrm{abc}$ \\
\hline Shockwave & $9.29 \mathrm{a}$ & $7.22 \mathrm{abc}$ & $0.01929 \mathrm{a}$ & $0.03876 \mathrm{bcd}$ & $372.58 \mathrm{a}$ & $91.86 \mathrm{bc}$ \\
\hline Sugar Cube & $9.38 \mathrm{a}$ & $6.78 \mathrm{~d}$ & $0.02347 \mathrm{a}$ & $0.04849 \mathrm{~b}$ & $331.34 \mathrm{a}$ & $92.20 \mathrm{bc}$ \\
\hline Sun Blushed & $10.64 \mathrm{a}$ & $6.77 \mathrm{~d}$ & $0.02746 \mathrm{a}$ & $0.08127 \mathrm{a}$ & 299.77 a & $91.10 \mathrm{c}$ \\
\hline Tirreno & $10.77 \mathrm{a}$ & $7.27 \mathrm{ab}$ & $0.01671 \mathrm{a}$ & $0.02855 \mathrm{~cd}$ & $591.99 a$ & $92.44 \mathrm{abc}$ \\
\hline Verona & $8.89 a$ & $6.98 \mathrm{a}-\mathrm{d}$ & $0.02512 \mathrm{a}$ & $0.04021 \mathrm{bc}$ & $288.31 \mathrm{a}$ & $92.50 \mathrm{abc}$ \\
\hline Year & SSC (\%) & Flesh pH & \multicolumn{2}{|c|}{ TA $(\%)$} & $\mathrm{SSC} / \mathrm{TA}$ & $\begin{array}{c}\text { Juiciness ( } \% \\
\text { water content) }\end{array}$ \\
\hline 2018 & $8.58 \mathrm{~b}$ & $7.22 \mathrm{a}$ & \multicolumn{2}{|c|}{$0.02448 \mathrm{~b}$} & $413.18 \mathrm{a}$ & $92.08 \mathrm{~b}$ \\
\hline 2019 & $10.42 \mathrm{a}$ & $6.76 \mathrm{~b}$ & \multicolumn{2}{|c|}{$0.03922 \mathrm{a}$} & $293.54 b$ & $93.06 \mathrm{a}$ \\
\hline
\end{tabular}

${ }^{\mathrm{z}}$ TA calculated as percentage of malic acid. ${ }^{\mathrm{y}}$ SSC/TA is the ratio of soluble solids content to titratable acidity. ${ }^{\mathrm{x}}$ Values are the mean of four replications; data were analyzed using the mixed procedure and means were separated using PDIFF; values followed by different letters within a column are significantly different at $P<0.05$; "Aphrodite" was considered the standard.

Table 10. Rainfall amount (centimeters) and temperature (degrees Celsius) at three sites of muskmelon cultivar evaluation in Pennsylvania.

\begin{tabular}{ccccccccccccc}
\hline & \multicolumn{3}{c}{ Central Pennsylvania $^{z}$} & \multicolumn{3}{c}{ Southeastern Pennsylvania ${ }^{y}$} & \multicolumn{3}{c}{ Western Pennsylvania $^{\mathbf{x}}$} \\
\hline & $\mathbf{2 0 1 8}$ & $\mathbf{2 0 1 9}$ & $\begin{array}{c}\text { Mean } \\
\text { Rainfall }\end{array}$ & $\begin{array}{c}\text { Mean } \\
\text { Temp. }\end{array}$ & $\mathbf{2 0 1 8}$ & $\mathbf{2 0 1 9}$ & $\begin{array}{c}\text { Mean } \\
\text { Rainfall }\end{array}$ & $\begin{array}{c}\text { Mean } \\
\text { Temp. }\end{array}$ & $\mathbf{2 0 1 8}$ & $\begin{array}{c}\text { 2019 } \\
\text { Mean } \\
\text { Rainfall }\end{array}$ & $\begin{array}{c}\text { Mean } \\
\text { Temp. }\end{array}$ \\
\hline May & 10.59 & 15.80 & 8.79 & 15.28 & 13.08 & 16.94 & 10.21 & 16.50 & 13.92 & 22.12 & 11.07 & 14.50 \\
June & 13.72 & 9.17 & 10.44 & 20.17 & 12.24 & 13.18 & 10.26 & 21.56 & 17.73 & 10.90 & 11.66 & 19.11 \\
July & 23.80 & 6.38 & 8.94 & 22.28 & 24.71 & 13.59 & 11.81 & 23.44 & 21.84 & 10.24 & 12.47 & 21.28 \\
Aug. & 18.72 & 6.71 & 9.75 & 21.44 & 29.95 & 13.11 & 9.04 & 22.50 & 10.92 & 8.81 & 10.41 & 20.72 \\
Sept. & 22.83 & 4.75 & 9.07 & 17.06 & missing & 5.18 & 10.77 & 18.56 & 29.85 & 8.51 & 10.03 & 16.78 \\
\hline
\end{tabular}

${ }^{\mathrm{z}}$ Central site data collected from weather station $11.52 \mathrm{~km}$ from experimental plots in State College, PA [39]. ${ }^{\text {y }}$ Southeastern site data collected from an on-site weather station in Manheim, PA [39]. ${ }^{\times}$Western site data collected from weather station $15 \mathrm{~km}$ from experimental plots in Indiana, PA [40].

Low TA has been associated with high overall eating quality [14]. TA ranged between $0.01468 \%$ and $0.03448 \%$ in 2018 and between $0.02173 \%$ and $0.08127 \%$ in 2019 (Table 9). In 2018, no significant differences existed between cultivars. In 2019, "Aphrodite/Flexifort" 
had a lower TA than "Carousel", "Minerva", "Sugar Cube", "Sun Blushed", and "Verona". All other cultivars did not differ from "Aphrodite/Flexifort". Additionally, TA was higher in 2019 than in 2018. Irrigation and fertility management practices affect TA [41]. Each year of our study these factors were uniform for all cultivars. However, the amount of water supplied varied between years, owing to excessive rainfall in 2018 (Table 10). In a review, Etieene et al. [41] reported that higher amounts of water resulted in lower $\mathrm{pH}$ in several experiments and higher $\mathrm{pH}$ in others; although, none of these experiments focused on muskmelon. Our study suggested that higher amounts of water may result in a lower TA for muskmelon. More research is needed to verify this theory.

Flesh $\mathrm{pH}$ has been positively correlated with overall eating quality [14]. It ranged from 6.77 for "Sun Blushed" to 7.33 for "Aphrodite/Flexifort" (Table 9). "Aphrodite/Flexifort" had higher $\mathrm{pH}$ than "Astound", "Athena", "Carousel", "Ariel", "Sugar Cube", and "Sun Blushed". Additionally, it was higher in 2018 than in 2019. For muskmelon, it has been observed that $\mathrm{pH}$ is inversely related to TA, considering that a higher content of organic acids has been generally associated with a lower $\mathrm{pH}$ and vice versa $[14,42]$.

Soluble solids concentration alone is not a predictable measure of muskmelon quality as other factors are also contributors [43]. The ratio of SSC to TA (SSC/TA) has been more highly linked to consumer preference than SSC or TA alone [44]. In our study, SSC/TA ranged from 243.61 to 591.99 and was not different between cultivars (Table 9). It was higher in 2018 than in 2019. Considering the non-significant variation of the SSC between cultivars and the non-significant cultivar $\times$ year interaction, the variation of the SSC/TA ration over the two seasons was primarily due to the lower levels of TA observed in the first growing season, likely as a consequence of the high level of rainfall recorded in proximity of the harvest.

Consumer preference for muskmelon has been associated with high SSC and $\mathrm{pH}$, and low TA [14]. "Tirreno" and "Atlantis" met these criteria, as well as "Sugar Cube" and "Sun Blushed", whereas "Ariel" did not perform as well (Table 9). "Aphrodite/Flexifort" also met these criteria. Interestingly, non-grafted "Aphrodite" and "Aphrodite/RS841" did not. Grafting has improved muskmelon fruit quality by increasing photosynthesis and carbohydrate metabolism [45] and extending post-harvest life [46]. However, the rootstock and scion should be deliberately selected as negative effects can also occur, as shown for some cucurbits [47]. "Aphrodite/Flexifort" may be a better combination of muskmelon compared to "Aphrodite/RS841" based on the physicochemical traits measured in this study. Including "Aphrodite/Flexifort", "Tirreno", and "Atlantis" in a taste panel would be a next step, to determine how well consumer preference is correlated with their SSC, $\mathrm{pH}$, and TA values.

Percentage water content was recorded as a measure of juiciness. It ranged between 94.65\% to $91.10 \%$ and was higher in 2019 (93.06\%) than in 2018 (92.08\%) (Table 9). The percent water content in "Ariel" was higher than "Accolade", "Shockwave", "Sugar Cube", "Athena", and "Sun Blushed". All other cultivars did not differ from "Ariel". Despite these differences between cultivars, the range was relatively small, indicating they may all have had suitable juiciness.

\section{Conclusions}

Most cultivars did not produce different yields than "Aphrodite". One exception was "Sugar Cube" which produced a larger number of smaller-sized fruit than "Aphrodite" in four site-years. In the physicochemical evaluation, "Sugar Cube's" values for SSC, $\mathrm{pH}$, and TA combined considering all cultivars were inferior, indicating that consumer preference may be greater for other cultivars. Alternatively, the novelty of being a smallsized muskmelon may be more important to consumers than relative taste and could be further studied through consumer preference research.

"Infinite Gold", "Fuji", and "Shockwave" produced fruit with a lower mean marketable weight than "Aphrodite". Additionally, "Sun Blushed" and "Ariel" did not perform 
as well as other cultivars for SSC, $\mathrm{pH}$, and TA combined. "Aphrodite" may be preferred over these cultivars, depending on other factors, including market and farmer preference.

The yield between grafted and non-grafted "Aphrodite" was not different; however, biotic and abiotic stresses were minimal or absent throughout the study. Combined SSC, $\mathrm{pH}$, and TA values indicated that "Aphrodite/Flexifort" may be preferred by consumers compared to "Aphrodite/RS841" and non-grafted "Aphrodite". The "Aphrodite/Flexifort" combination offers an advantage for grafting, even in the absence of environmental stressors. "Sugar Cube" produced a unique, small-sized fruit that lends it to niche marketing. Small-sized fruit may suit the preferences of small households, which constitute a growing market segment. However, this cultivar had inferior combined SSC, $\mathrm{pH}$, and TA values compared to other cultivars. Evaluating "Sugar Cube" on a "Flexifort" rootstock may improve these physicochemical values.

Supplementary Materials: The following are available online at https:/ /www.mdpi.com/article/10 $.3390 /$ horticulturae7040069/s1, Table S1: $P$-values for cultivar, year, and the interaction between year and cultivar for fruit yield components measured in an evaluation of 20 hybrid cultivars and two grafted entries of muskmelon in 2018 and 2019 at three sites in Pennsylvania and Table S2: $P$-values for cultivar, year, and the interaction between year and cultivar for fruit physicochemical components measured in an evaluation of 14 hybrid cultivars and two grafted entries of muskmelon in 2018 and 2019 at the center site in Pennsylvania.

Author Contributions: Conceptualization, E.S., R.P., T.E., T.B. and F.D.; methodology, E.S., R.P., T.E., T.B. and F.D.; formal analysis, E.S.; investigation, E.S., R.P., T.E., T.B. and F.D.; writing-original draft preparation, E.S.; writing - review and editing, E.S., R.P., T.E., T.B., and F.D.; funding acquisition, E.S., R.P., T.E., T.B. and F.D.; All authors have read and agreed to the published version of the manuscript.

Funding: This research was funded by the Pennsylvania Vegetable Growers Association and Pennsylvania Vegetable Marketing and Research Program and in part was supported by the National Institute of Food and Agriculture, U.S. Department of Agriculture, Specialty Crops Research Initiative under award Number 2016-51181-25404. The USDA National Institute of Food and Agriculture and Hatch Appropriations under Project also supported the contribution of F. Di Gioia \#PEN04723 and Accession \#1020664.

Data Availability Statement: The data presented in this study are available on request from the corresponding author.

Acknowledgments: The authors would like to thank Yarnick's Farm, LLC in Indiana, Pennsylvania for hosting this study on their farm, Clifton Seed Co., Rupp Seeds, Sakata, Seedway LLC, Seminis, and Syngenta for providing the seed used in this study, Tri-Hishtil for providing grafted plants, Rich Marini for consulting on the statistical analysis used in this research, and Harini Shanker, Trevor Johnson, and Chris Sánchez for assisting with field and lab work.

Conflicts of Interest: The authors declare no conflict of interest. The funders had no role in the design of the study; in the collection, analyses, or interpretation of data; in the writing of the manuscript, or in the decision to publish the results.

\section{References}

1. United States Department of Agriculture. 2017 Census of Agriculture. Table 28. Vegetables, Potatoes, and Melons Harvested for Sale: 2017 and 2012. Available online: https:/ /www.nass.U.S.D.A..gov/Publications/AgCensus/2017/Full_Report/Volume_1, _Chapter_2_US_State_Level/st99_2_0029_0029.pdf (accessed on 2 February 2021).

2. Peters, C.J.; Gómez, M.I.; Griffin, T. Roles of regional production in a global food system. Renew. Agric. Food Syst. 2019, 1-11. [CrossRef]

3. Williams, T.V.; Roberts, W. Is vegetable variety evaluation and reporting becoming a lost art? An industry perspective. HortTechnology 2002, 12, 553-559. [CrossRef]

4. Butzler, T.; (Pennsylvania State Extension, Lock Haven, PA, USA). Personal communication, 5 February 2019.

5. Kubota, C.; McClure, M.A.; Kokalis-Burelle, N.; Bausher, M.G.; Rosskopf, E.N. Vegetable grafting: History, use and current technology status in North America. HortScience 2008, 43, 1664-1669. [CrossRef]

6. Davis, A.R.; Perkins-Veazie, P.; Sakata, Y.; López-Galarza, S.; Maroto, J.V.; Lee, S.; Huh, Y.; Sun, Z.; Miguel, A.; King, S.R. Cucurbit grafting. Crit. Rev. Plant Sci. 2008, 27, 50-74. [CrossRef] 
7. Rouphael, Y.; Cardarelli, M.; Rea, E.; Colla, G. Improving melon and cucumber photosynthetic activity, mineral composition, and growth performance under salinity stress by grafting onto Cucurbita hybrid rootstocks. Photosynthetica 2012, 50, 180-188. [CrossRef]

8. Di Gioia, F.; Serio, F.; Buttaro, D.; Ayala, O.; Santamaria, P. Influence of rootstock on vegetative growth, fruit yield and quality in 'Cuore di Bue', an heirloom tomato. J. Hort. Sci. Biotech. 2010, 85, 477-482. [CrossRef]

9. Di Gioia, F.; Aprile, A.; Sabella, E.; Santamaria, P.; Pardossi, A.; Miceli, A.; De Bellis, L.; Nutricati, E. Grafting response to excess boron and expression analysis of genes coding boron transporters in tomato. Plant Biol. 2017, 19, 728-735. [CrossRef] [PubMed]

10. Schwarz, D.; Rouphael, Y.; Colla, G.; Venema, J.H. Grafting as a tool to improve tolerance of vegetables to abiotic stresses: Thermal stress, water stress and organic pollutants. Sci. Hortic. 2010, 127, 162-171. [CrossRef]

11. Kyriacou, M.C.; Rouphael, Y.; Colla, G.; Zrenner, R.; Schwarz, D. Vegetable grafting: The implications of a growing agronomic imperative for vegetable fruit quality and nutritive value. Front. Plant Sci. 2017, 8, 741. [CrossRef] [PubMed]

12. Verzera, A.; Dima, G.; Tripodi, G.; Condurso, C.; Crino, P.; Romano, D.; Mazzaglia, A.; Lanza, C.M.; Restuccia, C.; Paratore, A. Aroma and sensory quality of honeydew melon fruits (Cucumis melo L. subsp. melo var. inodorus H. Jacq.) in relation to different rootstocks. Sci. Hortic. 2014, 169, 118-124. [CrossRef]

13. Lester, G. Consumer preference quality attributes of melon fruits. Acta Hortic. 2006, 712, 175-182. [CrossRef]

14. Park, E.; Luo, Y.; Marine, S.C.; Everts, K.L.; Micallef, S.A.; Bolten, S.; Stommel, J. Consumer preference and physiochemical evaluation of organically grown melons. Postharvest Biol. Technol. 2018, 141, 77-85. [CrossRef]

15. Butzler, T.M.; Sánchez, E.S.; Bogash, S.M.; Elkner, T.E.; Lamont, W.J., Jr.; Pollock RStivers, L.J. Pennsylvania statewide sweet corn cultivar evaluation. HortTechnology 2015, 25, 687-695. [CrossRef]

16. Butzler, T.; Sánchez, E.S.; Elkner, T.E.; Lamont, W., Jr.; Pollock, R. Yield and handle quality of twenty-one carving pumpkin varieties. J. NACAA 2020, 13, 2158-9429.

17. Sánchez, E.S.; Butzler, T.M.; Bogash, S.M.; Elkner, T.E.; Oesterling, R.E.; Orzolek, M.D.; Stivers, L.J. Pennsylvania statewide bell pepper cultivar evaluation. HortTechnology 2011, 21, 384-390. [CrossRef]

18. Sánchez, E.S.; Butzler, T.M.; Stivers, L.J.; Elkner, T.E.; Bogash, S.M.; Oesterling, R.E.; Orzolek, M.D. Pennsylvania statewide winter squash evaluation. HortTechnology 2012, 22, 826-837. [CrossRef]

19. Sánchez, E.; Butzler, T.; Stivers, L.; Pollock, R.; Elkner, T.; Bogash SLamont, W. Performance of spring and fall broccoli cultivars for the mid-Atlantic region. HortTechnology 2016, 26, 869-876. [CrossRef]

20. Wyenandt, C.A.; van Vuuren, M.M.I. 2018 Mid-Atlantic Commercial Vegetable Production Recommendations. Penn State Extension Publication, University Part, PA \#AGRS-028G. Available online: https://www.sites.ext.vt.edu/newsletter-archive/comm-vegprod-recommendations/2018.pdf (accessed on 3 February 2021).

21. Osborne, J.; Simonne, E. Data collection and statistical topics for the preparation and review of manuscripts. HortTechnology 2002, 12, 567-583. [CrossRef]

22. Lilley, J.M.; Sánchez, E.S. The potential of strip tillage for organic cucurbit production. HortTechnology 2016, 26, 628-636. [CrossRef]

23. Sánchez, E.S.; Hernández, E.; Gleason, M.L.; Batzer, J.C.; Williams, M.A.; Coolong, T.; Bessin, R. Optimizing Row Cover Deployment for Managing Bacterial Wilt and Using Compost for Organic Muskmelon Production. HortTechnology 2015, 25, 762-768. [CrossRef]

24. Nair, A.; Love, F. In Search of the Perfect Muskmelon. 2019. Available online: https://www.news.iastate.edu/news/2019/08/22 / muskmelon (accessed on 2 February 2021).

25. Louws, F.J.; Rivard, C.L.; Kubota, C. Grafting fruiting vegetables to manage soilborne pathogens, foliar pathogens, arthropods, and weeds. Sci. Hortic. 2010, 127, 127-146. [CrossRef]

26. Di Gioia, F.; Signore, A.; Serio, F.; Santamaria, P. Grafting improves tomato salinity tolerance through sodium partitioning within the shoot. HortScience 2013, 48, 855-862. [CrossRef]

27. López-Serrano, L.; Canet-Sanchis, G.; Selak, G.V.; Penella, C.; San Bautista, A.; López-Galarza, S.; Calatayud, Á. Pepper rootstock and scion physiological responses under drought stress. Front. Plant Sci. 2019, 10. [CrossRef] [PubMed]

28. Rouphael, Y.; Kyriacou, M.C.; Colla, G. Vegetable grafting: A toolbox for securing yield stability under multiple stress conditions. Front. Plant Sci. 2018, 8, 2255. [CrossRef]

29. Kleinhenz, M.D. Description of Commercial Rootstocks as of February 5 2015. Available online: https://hos.ifas.ufl.edu/media/ hosifasufledu/documents / Description-of-Commercial-Cucurbit-Rootstocks-as-of-February-5,-2015.pdf (accessed on 3 February 2021).

30. Flexifort. Available online: https://www.enzazaden.com/us/products-and-services/our-products/Rootstock/Flexifort (accessed on 3 February 2021).

31. Colla, G.; Rouphael, Y.; Cardarelli, M.; Massa, D.; Rea, E. Yield, fruit quality and mineral composition of grafted melon plants grown under saline conditions. J. Hort. Sci. Biotech. 2006, 81, 146-152. [CrossRef]

32. Crino, P.; Bianco, C.L.; Rouphael, Y.; Colla, G.; Saccardo, F.; Paratore, A. Evaluation of rootstock resistance to fusarium wilt and gummy stem blight and effect on yield and quality of a grafted 'Inodorus' melon. HortScience 2007, 42, 521-525. [CrossRef]

33. Edelstein, M.; Ben-Hur, M.; Cohen, R.; Burger, Y.; Ravina, I. Boron and salinity effects on grafted and non-grafted melon plants. Plant Soil 2005, 269, 273-284. [CrossRef]

34. Ruiz, J.M.; Belakbir, A.; López-Cantarero, I.; Romero, L. Leaf-macronutrient content and yield in grafted melon plants. A model to evaluate the influence of rootstock genotype. Sci. Hortic. 1997, 71, 227-234. [CrossRef] 
35. United States Department of Agriculture (U.S.D.A.). Cantaloupes, Honeydew, Honey Ball and Other Similar Melons. Shipping Point and Market Inspection Instructions, ed. Available online: https://www.ams.usda.gov/sites/default/files/media/ Honeydew_Inspection_Instructions\%5B1\%5D.pdf (accessed on 3 February 2021).

36. Fabeiro, C.; Martin de Santa Olalla, F.; de Juan, J.A. Production of muskmelon (Cucumis melo L.) under controlled deficit irrigation in a semi-arid climate. Agric. Water Manag. 2002, 54, 93-105. [CrossRef]

37. Wells, J.A.; Nugnet, P.E. Effect of high soil moisture on quality of muskmelon. HortScience 1980, 15, $258-259$.

38. National and Oceanic and Atmospheric Administration. 2018 in Context: Record Precipitation. Available online: https: / / www.weather.gov/ctp/RecordPrecip2018 (accessed on 3 February 2021).

39. Nationa Weather Service Forcast Office, State College, PA, Eastern Site-Landisville 2 NW, PA, Central Site-State College, PA. 2018. Available online: https://w2.weather.gov/climate/xmacis.php?wfo=ctp (accessed on 16 March 2021).

40. National Weather Service Forcast Office, Pittsburgh, PA, USA, Western Site-Indiana 3 Se, PA. 2018. Available online: https: //w2.weather.gov / climate/xmacis.php?wfo=pbz (accessed on 16 March 2021).

41. Etienne, A.; Génard, M.; Lobit, P.; Mbeguié-A-Mbéguie, D.; Bugaud, C. What controls fleshy fruit acitidty? A review of malate and citrate accumulation in fruit cells. J. Exp. Bot. 2013, 64, 1451-1469. [CrossRef] [PubMed]

42. Simandjuntak, V.; Barrett, D.M.; Wrolstad, R.E. Cultivar and maturity effects on muskmelon (Cucumis melo) colour, texture and cell wall polysaccharide composition. J. Sci. Food Agric. 1996, 71, 282-290. [CrossRef]

43. Lester, G.; Shellie, K.C. Postharvest sensory and physicochemical attributes of Honey dew melon fruits. HortScience 1992, 27, 1012-1014. [CrossRef]

44. Albuquerque, B.; Lindon, F.C.; Graca Barreiro, M. A case study on the flavor properties of melon (Cucumis melo L.) cultivars. Fruits 2006, 61, 333-339. [CrossRef]

45. Liu, Y.; Qi, H.; Bai, C.; Qi, M.; Xu, C.; Hao, J.; Li, Y.; Li, T. Grafting helps improve photosynthesis and carbohydrate metabolism in leaves of muskmelon. Int. J. Bio. Sci. 2011, 7, 1161-1170. [CrossRef]

46. Zhao, X.; Guo, Y.; Huber, D.L.; Lee, J. Grafting effects on postharvest ripening and quality of 1-methylcyclopropene-treated muskmelon fruit. Sci. Hortic. 2011, 130, 581-587. [CrossRef]

47. Davis, A.R.; Hassell, R.; Levi, A.; King, S.R.; Zhang, X. Grafting effects on vegetable quality. HortScience 2008, 43, 1670-1672. [CrossRef] 